\title{
Patients who attempted suicide emphasised they wanted to be well cared for and receive understanding and confirmation
}

Samuelsson M, Wiklander M, Asberg M, et al. Psychiatric care as seen by the attempted suicide patient.J Adv Nurs 2000 Sep;32:635-43.

\section{QUESTION: What are the experiences of patients during inpatient psychiatric care after a suicide attempt?}

\section{Design}

Qualitative description using content analysis of interview data.

\section{Setting}

Specialised ward in the psychiatric clinic of the Karolinska Hospital, Stockholm, Sweden.

\section{Patients}

18 Swedish speaking patients (age range 18-53 y, 67\% men) who had recently attempted suicide.

\section{Methods}

The patients were interviewed as close to hospital discharge as possible. They were asked to describe their experiences of the care they received. Researchers used an interview guide to enquire about admission to hospital, feelings and reactions, and positive and negative experiences during the hospital stay. The interviews that lasted between 30 and 70 minutes were transcribed verbatim. A qualitative content analysis of the meanings, intentions, consequences and the context of data was done.

\section{Main findings}

3 central categories were identified: (1) being a psychiatric patient, (2) perceptions of the caregivers and the care provided, and (3) important aspects of the psychiatric care received. The patients described mixed feelings about being a psychiatric patient. They commonly reported feelings of discomfort, nervousness, failure, and shame as well as feelings of relief at being admitted to hospital because they could not cope any longer. Although the patients had difficulties accepting admission to hospital in a psychiatric clinic, they understood the need for it. Patients expressed shock and horror over having tried to kill themselves.

The patients described their perceptions of the caregivers and care provided in positive and negative ways. Many patients felt that the staff really cared and showed concern. The word "security" was used by more than half the patients. Other patients felt a lack of consideration and that no one seemed to care. These patients perceived the staff as indifferent and uncaring. The importance of being well cared for and experiencing understanding, confirmation, trust, and respect were emphasised. Lack of confirmation sometimes contributed to patients' feelings of being burdensome, demands for discharge, or even another suicide attempt.

Important aspects of the psychiatric care received included the opportunity to talk to staff members and be understood, the invitation by staff to access the ward after discharge if necessary, and the admission to hospital itself, which patients viewed as life saving.

\section{Conclusion}

Patients who had attempted suicide and were inpatients on a psychiatric ward emphasised that they wanted to be well cared for and receive understanding and confirmation.

\section{COMMENTARY}

This study by Samuelsson et al provides fascinating insight into an important and under researched area of psychiatric care. The findings resonate with the broader literature on professional-patient relationships, particularly the importance of being patient centred. ${ }^{1}$ Although this suggests the findings may be generalisable beyond the setting of a specialised secondary care ward in Sweden, it would be important to first learn more about the special training the nurses received to provide care for suicidal patients.

There were more positive than negative descriptions of caregivers; however, some patients perceived a lack of consideration and respect from the psychiatric staff, which included nurses, psychiatrists, a social worker, and a psychologist*. Samuelsson et al suggest that this might be a protective mechanism used by the staff to distance themselves from emotional aspects of their work. A less than positive disposition may, however, also reflect the tension created by such patients who do not conform to the traditional sick role, a reaction that is well recognised and documented in the healthcare literature. ${ }^{2}$

Samuelsson et al suggest 3 strategies to help nurses who work with patients who have attempted suicide: (1) continual professional clinical supervision to increase their skills in dealing with individual patients, increase their coping skills, and help them to defuse their own negative emotional reactions; (2) adequate knowledge of suicidology; and (3) individualised care plans that facilitate nurse-patient collaboration in treatment. Study findings may also suggest the need for psychiatric staff to participate in inservice sessions in which they can examine their own attitudes towards patients who attempt suicide.

This study emphasises the need for further hypothesis generating research to explore the particular characteristics of patients and settings that determine satisfaction with care and caregivers.

Helen Lester, MB, BCH, MD Senior Lecturer in Primary Care The Medical School, University of Birmingham Birmingham, $U K$

*Information provided by the authors.

1 Little P, Everitt H, Williamson I, et al. Preferences of patients for patient centred approach to consultation in primary care: observational study. BMJ 2001;322:468-73.

2 Jeffrey R. Normal rubbish: deviant patients in casualty departments. Sociol Health Iln 1979;1:90-107.

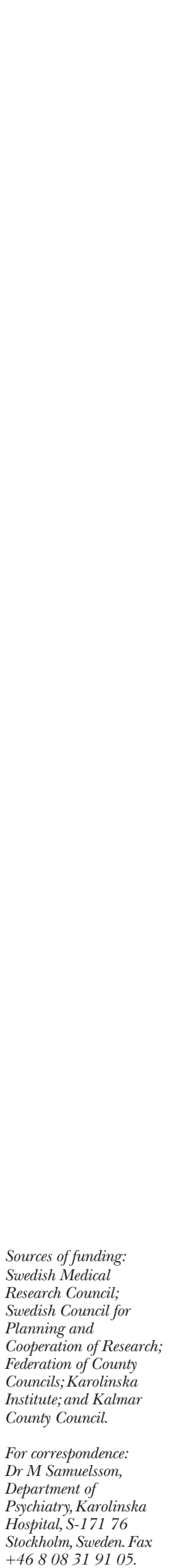

